RESPIRATORY INFECTION

\title{
Does influenza vaccination increase consultations, corticosteroid prescriptions, or exacerbations in subjects with asthma or chronic obstructive pulmonary disease?
}

\author{
L J Tata, J West, T Harrison, P Farrington, C Smith, R Hubbard
}

Thorax 2003;58:835-839

See end of article for authors' affiliations

\section{Correspondence to:} Ms L J Tata, Division of Epidemiology and Public Health, University of

Nottingham, Nottingham, UK; laila.tata@nottingham. ac.uk

Revised version received 7 May 2003

Accepted for publication

7 July 2003
Background: Concern over the safety of influenza vaccination in individuals with obstructive airways disease has contributed to suboptimal rates of vaccine uptake in this group. We investigated the safety of influenza vaccine in older people with asthma or chronic obstructive pulmonary disease (COPD) in a cohort from the UK General Practice Research Database (GPRD).

Methods: A population based cohort study of 12000 individuals with asthma or COPD from 432 general practices was conducted. Incidence rate ratios (IRR) were calculated for asthma or COPD diagnoses, prescriptions for oral corticosteroids, and acute exacerbations on the day of vaccination and on days 1-2 and 3-14 after vaccination compared with other time periods in the influenza season.

Results: The IRRs for asthma or COPD diagnoses and oral corticosteroid prescriptions were increased on the day of vaccination (for example, the IRR for oral corticosteroid prescriptions for subjects with asthma during the 1992-3 influenza season was 8.24 (95\% confidence interval 5.54 to 12.26$)$ ). However, there was no consistent increase in the IRR of any of the outcomes on days 1-2 or 3-14 after vaccination, and most of these IRRs were close to 1. Rates of exacerbation were low and showed no consistent statistically significant increase during any risk periods.

Conclusions: Older people with asthma or COPD commonly have diagnoses recorded or prescriptions for oral corticosteroids given on the day of influenza vaccination, but there is no increased risk of adverse acute outcomes in the first 2 weeks after vaccination. Our findings strongly suggest that influenza vaccination is safe in this population. l nfluenza vaccination reduces hospital admissions and death due to influenza and other respiratory illnesses ${ }^{1-5}$ and is currently recommended for people with chronic pulmonary disorders. ${ }^{6}$ However, rates of uptake are not optimal, ${ }^{7}$ partly because of patient and physician concern that vaccination may induce acute exacerbations in those with asthma and chronic obstructive pulmonary disease (COPD)..$^{78}$

Well conducted clinical trials have found no excess risk of adverse respiratory effects associated with influenza vaccination in older people, but only a small proportion of populations studied had respiratory diseases. ${ }^{9}{ }^{10}$ One trial of influenza vaccine safety in adults aged under 65 years with asthma found no risk of exacerbation. ${ }^{11}$ Two other trials in older adults with asthma had similar findings, ${ }^{12}{ }^{13}$ but these two studies lacked sufficient statistical power to detect small but important increases in acute clinical exacerbations. The evidence base for vaccine safety in people with COPD is less robust and two recent systematic reviews concluded that further adequately powered studies were needed for both individuals with asthma and those with COPD. ${ }^{14}{ }^{15}$

We have analysed data from a cohort of 12000 subjects over 65 years of age with asthma or COPD drawn from the UK General Practice Research Database (GPRD). A selfcontrolled case series method was used to exclude confounding by disease severity and to determine whether influenza vaccination is associated with an increased incidence of recorded diagnoses of asthma or COPD, oral corticosteroid prescriptions, or acute exacerbations.

\section{METHODS}

The GPRD is the largest longitudinal primary care dataset in the world, comprising more than 8 million people in over 700 general practices across the UK. ${ }^{16}{ }^{17}$ Morbidity and prescribing data have consistently been found to have high accuracy, ${ }^{18}$ and the database has proved to be a good source of epidemiological data on respiratory diseases. ${ }^{17} 19$

Our cohort consisted of a random sample of 6000 people with asthma and 6000 with COPD (asthma status was mutually exclusive of COPD and vice versa) who were 6579 years of age on 1 January 1991. They were followed up until 15 February 1998. The influenza season was defined as 1 October to 30 April, and the association between vaccination and disease outcomes was investigated in the 1991-2, 1992-3, and 1993-4 seasons. For each influenza season we extracted patient records for influenza vaccinations, oral corticosteroid prescriptions, and all incident diagnoses of asthma or COPD. The GPRD diagnosis codes were identified using the Oxford Medical Indexing System (OXMIS) and Read Clinical Classification coding, which can be cross referenced to the International Classification of Diseases (code lists available from authors). We estimated the crude incidence rates of prescriptions and all diagnostic codes for the 6 months before and after vaccination using the median date of vaccination as an index date for the unvaccinated individuals. Crude incidence rates of acute exacerbations only were then estimated by extracting codes with the terms "acute", "attack", or "exacerbation" from the complete diagnostic code lists.

For our main analyses we used the case series technique (with GLIM version 4.09 software) which is a self-controlled method derived from the cohort model, specifically developed to investigate vaccine safety. ${ }^{20-22}$ This method estimates relative incidence in high risk compared with low risk periods, using data from vaccinated individuals who must have at least one outcome, so we restricted our analyses to 
Table 1 Rates of asthma and COPD related outcomes before and after vaccination and index dates in 1991-2

\begin{tabular}{|c|c|c|c|c|}
\hline \multirow[b]{3}{*}{ Outcome } & \multicolumn{4}{|c|}{ Rate (per 1000 person years) } \\
\hline & \multicolumn{2}{|l|}{$\begin{array}{l}\text { Vaccinated } \\
\text { ( } n=4652)\end{array}$} & \multicolumn{2}{|c|}{$\begin{array}{l}\text { Unvaccinated } \\
(\mathrm{n}=7348)\end{array}$} \\
\hline & $\begin{array}{l}6 \text { months } \\
\text { before* }\end{array}$ & $\begin{array}{l}6 \text { months } \\
\text { after* }\end{array}$ & $\begin{array}{l}6 \text { months } \\
\text { before }^{\star *}\end{array}$ & $\begin{array}{l}6 \text { months } \\
\text { after** }\end{array}$ \\
\hline $\begin{array}{l}\text { Any asthma diagnostic } \\
\text { code }\end{array}$ & 844 & 884 & 568 & 626 \\
\hline $\begin{array}{l}\text { Asthma exacerbation } \\
\text { code }\end{array}$ & 28 & 27 & 15 & 19 \\
\hline $\begin{array}{l}\text { Oral corticosteroid } \\
\text { prescription }\end{array}$ & 638 & 660 & 467 & 531 \\
\hline $\begin{array}{l}\text { Any COPD diagnostic } \\
\text { code }\end{array}$ & 470 & 597 & 325 & 406 \\
\hline $\begin{array}{l}\text { COPD exacerbation } \\
\text { code }\end{array}$ & 50 & 71 & 26 & 34 \\
\hline $\begin{array}{l}\text { Oral corticosteroid } \\
\text { prescription }\end{array}$ & 495 & 547 & 277 & 313 \\
\hline $\begin{array}{l}\text { *Vaccination day. } \\
\text { **October } 28 .\end{array}$ & & & & \\
\hline
\end{tabular}

these people. The main advantage of this method is the elimination of inter-individual confounding, particularly by disease severity. We examined three high risk periods: the day of vaccination, and 1-2 days and 3-14 days after vaccination. For each individual we compared incidence rates of prescriptions, all diagnosis codes, and acute exacerbations during the high risk periods with incidence rates during a baseline period bounded by the remaining influenza season. Using conditional Poisson regression for case-series analysis we estimated incidence rate ratios, adjusted for calendar time in months. We used a sample size calculation developed by Farrington et $a^{21}$ and estimated that, with 120 outcome events, we would have $90 \%$ power to detect at least a doubling of risk, assuming that $75 \%$ of our cohort was vaccinated.

\section{RESULTS}

From the original cohort we identified 2552 (43\%) individuals with asthma and 2100 (35\%) with COPD who received an influenza vaccination in 1991-2; 2349 (39\%) and 1855 (31\%) in 1992-3; and 2441 (41\%) and 1963 (33\%) in 1993-4. Sixty two percent of those with asthma and $37 \%$ with COPD were women, and the proportion of men vaccinated was similar to that of the women. Current or recent smokers accounted for $24 \%$ of those with asthma and $47 \%$ of those with COPD. Crude incidence rates for all three outcomes generally increased slightly in the 6 months after vaccination compared with the 6 months before vaccination in the 19912 influenza season (table 1). Using the approximate median vaccination day (28 October), our calculated outcome rates for unvaccinated individuals over similar time periods were consistently lower than those for vaccinated individuals, but showed the same increase in the second 6 month period. Rates were similar and showed analogous trends in the 19923 and 1993-4 seasons.

Of those vaccinated, only 696 (27\%) with asthma and 416 (20\%) with COPD had one or more diagnosis codes in 19912 , which was similar in the following seasons (table 2 ). Of these, $320(46 \%)$ with asthma and 152 (37\%) with COPD had more than one recorded outcome in 1991-2; 271 (44\%) and $124(37 \%)$ in 1992-3; and $294(46 \%)$ and $124(36 \%)$ in $1993-$ 4. Specific codes for acute exacerbations or oral corticosteroid prescriptions were less common than more general asthma or COPD diagnosis codes (table 2).

Figures 1 and 2 illustrate the distribution of the three outcomes for individuals who were vaccinated in the 1991-2 influenza season. There is a relatively even distribution of asthma and COPD diagnosis codes throughout the influenza season, with the exception of a sharp increase in reported codes on the day of vaccination. The same temporal clustering was seen for oral corticosteroid prescriptions, however, when we plotted exacerbations, but there was no evident increase on the day of vaccination nor during the period after vaccination. Plots were similar for outcomes in the other two seasons.

Table 2 Case series analysis of asthma and COPD related outcomes in defined risk periods after influenza vaccination

\begin{tabular}{|c|c|c|c|c|c|}
\hline \multirow[b]{2}{*}{ Outcome } & \multirow[b]{2}{*}{ No of cases } & \multirow{2}{*}{$\begin{array}{l}\text { No of } \\
\text { outcomes }\end{array}$} & \multicolumn{3}{|c|}{ Incidence rate ratio $(95 \% \mathrm{Cl})$} \\
\hline & & & Vaccination day & 1-2 days* & 3-14 days* \\
\hline \multicolumn{6}{|c|}{ Any asthma diagnostic code } \\
\hline 1991-1992 & 696 & 1385 & 14.93 (11.79 to 18.89$)$ & $0.76(0.39$ to 1.47$)$ & $0.88(0.67$ to 1.14$)$ \\
\hline 1992-1993 & 616 & 1173 & 14.31 (11.02 to 18.59$)$ & $0.61(0.27$ to 1.37$)$ & $1.25(0.98$ to 1.61$)$ \\
\hline 1993-1994 & 639 & 1239 & $12.12(9.33$ to 15.74$)$ & $1.16(0.67$ to 2.02$)$ & $0.96(0.74$ to 1.25$)$ \\
\hline \multicolumn{6}{|c|}{ Asthma exacerbation code } \\
\hline 1991-1992 & 38 & 44 & $4.27(0.56$ to 32.62$)$ & ** & 0.79 (0.18 to 3.49 ) \\
\hline 1992-1993 & 38 & 50 & ** & $1.99(0.26$ to 15.20$)$ & $2.32(0.95$ to 5.65$)$ \\
\hline 1993-1994 & 49 & 60 & ** & $1.61(0.21$ to 12.20$)$ & 1.69 (0.67 to 4.26$)$ \\
\hline \multicolumn{6}{|c|}{ Oral corticosteroid prescription } \\
\hline 1991-1992 & 352 & 971 & 5.01 (3.27 to 7.67$)$ & $0.87(0.43$ to 1.75$)$ & 0.74 (0.54 to 1.03$)$ \\
\hline $1992-1993$ & 329 & 824 & 8.24 (5.54 to 12.26$)$ & $1.04(0.49$ to 2.22$)$ & $1.18(0.87$ to 1.61$)$ \\
\hline 1993-1994 & 354 & 848 & 4.06 (2.49 to 6.64$)$ & $0.96(0.48$ to 1.94$)$ & $0.79(0.57$ to 1.11$)$ \\
\hline \multicolumn{6}{|c|}{ Any COPD diagnostic code } \\
\hline $1991-1992$ & 416 & 744 & $11.41(8.02$ to 16.25$)$ & $0.31(0.08$ to 1.24$)$ & 0.61 (0.40 to 0.93$)$ \\
\hline $1992-1993$ & 339 & 599 & $14.23(9.86$ to 20.53$)$ & $0.58(0.18$ to 1.80$)$ & 1.00 (0.68 to 1.48$)$ \\
\hline 1993-1994 & 345 & 632 & 7.38 (4.62 to 11.78$)$ & 0.55 (0.18 to 1.73$)$ & 1.07 (0.75 to 1.53$)$ \\
\hline \multicolumn{6}{|c|}{ COPD exacerbation code } \\
\hline 1991-1992 & 61 & 86 & $2.25(0.30$ to 16.88$)$ & ** & 0.19 (0.03 to 1.42$)$ \\
\hline 1992-1993 & 62 & 89 & $16.86(6.16$ to 46.15$)$ & ** & $0.74(0.22$ to 2.48$)$ \\
\hline 1993-1994 & 68 & 90 & $2.63(0.35$ to 19.64$)$ & ** & $1.20(0.49$ to 2.92$)$ \\
\hline \multicolumn{6}{|c|}{ Oral corticosteroid prescription } \\
\hline 1991-1992 & 238 & 676 & 5.41 (3.28 to 8.92$)$ & $0.32(0.08$ to 1.27$)$ & $0.81(0.55$ to 1.18$)$ \\
\hline 1992-1993 & 197 & 541 & 7.47 (4.62 to 12.06$)$ & $0.98(0.40$ to 2.38$)$ & 1.09 (0.75 to 1.58$)$ \\
\hline 1993-1994 & 213 & 560 & 2.91 (1.43 to 5.91$)$ & $0.18(0.03$ to 1.29$)$ & $0.82(0.55$ to 1.22$)$ \\
\hline
\end{tabular}



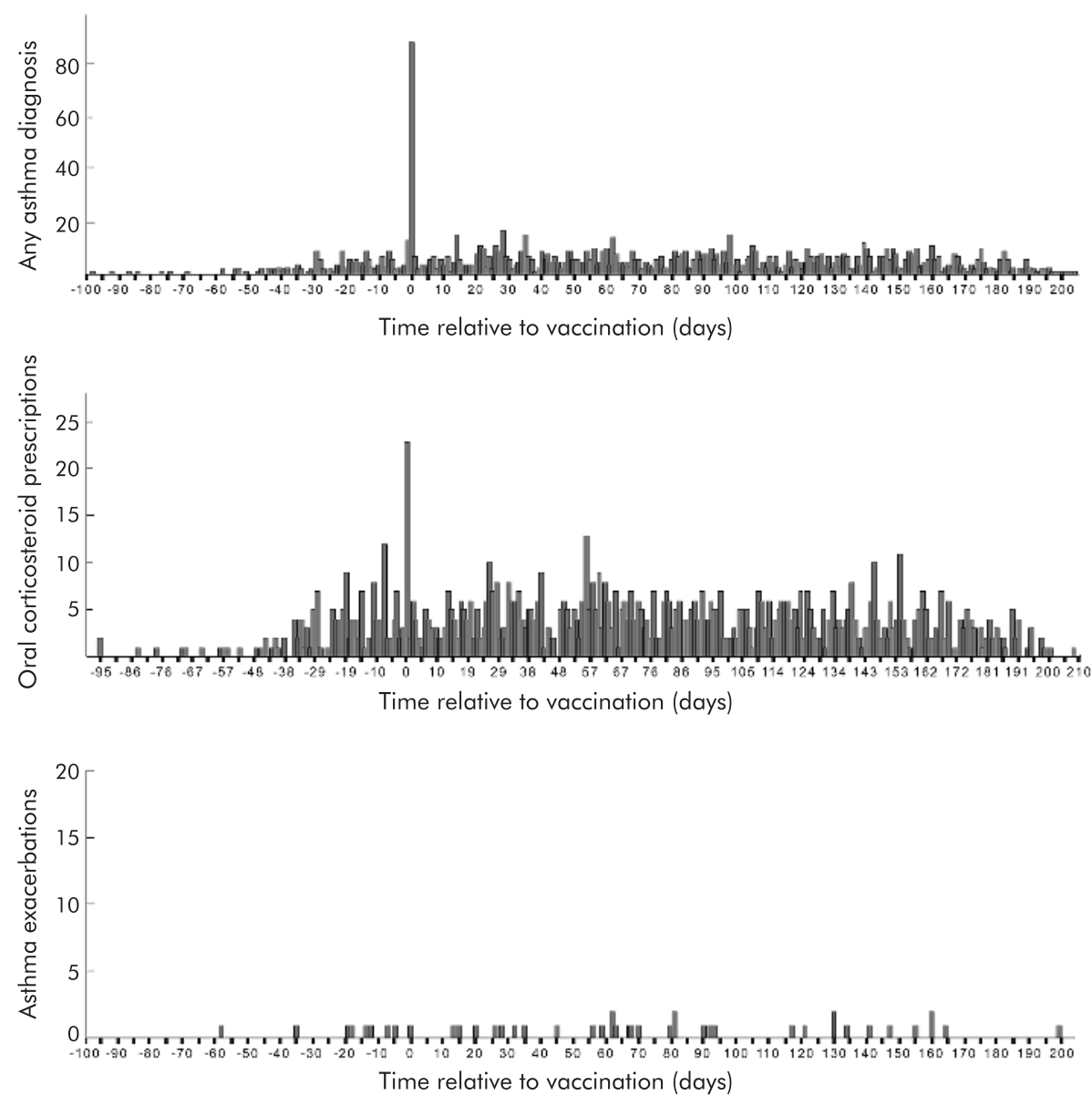

Figure 1 Frequency of all asthma diagnostic codes, oral corticosteroid prescriptions, and acute exacerbations in relation to the day of vaccination: 1991-2 season.

The relative incidence of asthma and COPD diagnoses and of oral corticosteroid prescriptions estimated using the case series method increased significantly on the day of vaccination with most rate ratios greater than 5.0 for each influenza season (table 2). However, at 1-2 and 3-14 days after vaccination the rate ratios for diagnoses and prescriptions were close to unity and none showed statistically significant effects. Restricting our analysis to exacerbation codes, the incidence of asthma exacerbations did not show a statistically significant increase on vaccination day, nor $1-2$ days or 314 days after vaccination. Exacerbations of COPD in 1992-3 significantly increased on the day of vaccination but this was not the case in the other two seasons. Incidence rate ratios in the 1-2 day and 3-14 day periods after vaccination indicated that COPD exacerbations did not increase significantly.

\section{DISCUSSION}

In this large population based sample of 12000 older people with asthma and COPD, an increase in the recording of asthma and COPD diagnoses and oral corticosteroid prescriptions occurred on the day of the influenza vaccination but no increases were found 1-2 days or 3-14 days after vaccination. Our explanation for these findings is that attending for vaccination provides an opportunity for the general practitioner to record chronic disease diagnosesparticularly if they are the reason why the vaccination has been recommended-and to prescribe courses of corticosteroids which may be kept at home in anticipation of possible acute exacerbations later in the season. If a true adverse impact of the vaccine was present, we would expect to see an increase in the recorded events in the days after vaccination, and the lack of increase in events during this period is reassuring. While it is possible that low exacerbation rates in the days following vaccination may partially reflect a reduced need to return to the general practitioner if a patient was prescribed a corticosteroid on the day of vaccination, it is unlikely to explain these reductions in risk since only a few of those vaccinated received a prescription on the same day $(<1 \%)$, and individuals with exacerbations may well still contact their general practitioner. If there was a true increase in exacerbations on the day of vaccination, we would expect consistently high recording of these acute events in the GPRD across all three seasons in concurrence with the recording of the vaccination, so a single significantly raised incidence of COPD exacerbations on the day of vaccination in the 1992-3 season should not be overinterpreted. Our results therefore strongly suggest that vaccination does not have a deleterious impact on asthma or COPD control.

Vaccination coverage in our cohort was low (40\%) and did not meet our original assumption of $75 \%$, which therefore reduced the statistical power of the study. A recalculation, however, indicated that with 231 outcome events we would still have $90 \%$ power to detect at least doubling of risk with only $40 \%$ vaccination coverage, so our study still had sufficient statistical power for our analyses of all diagnosis codes and prescriptions. Exacerbation codes accounted for less than $5 \%$ of all asthma diagnosis codes, and less than $15 \%$ of all COPD diagnosis codes in our main analysis, which will 

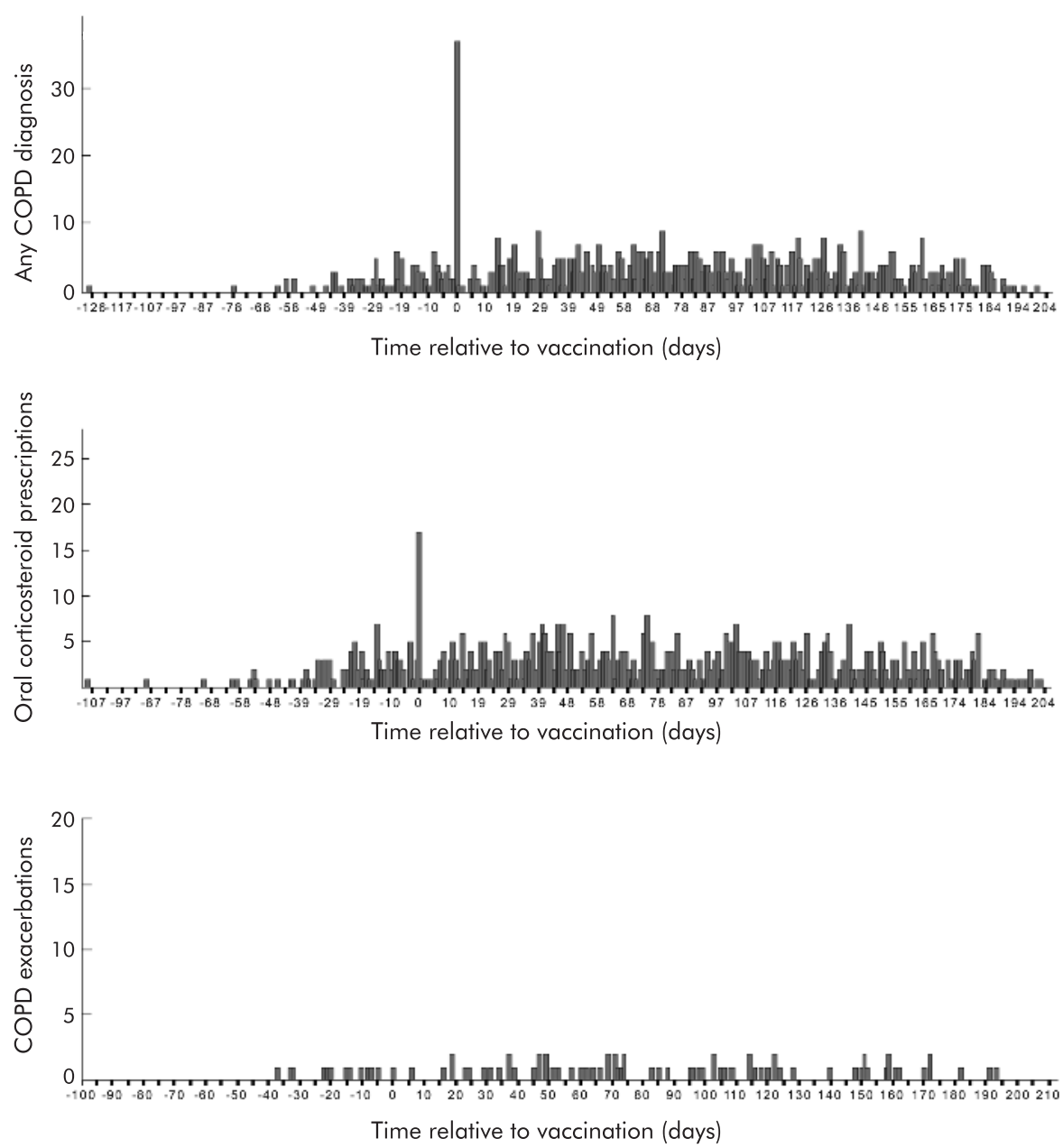

Figure 2 Frequency of COPD diagnostic codes, oral corticosteroid prescriptions, and acute exacerbations in relation to the day of vaccination: $1991-2$ season.

have reduced power for this analysis. However, rates of exacerbation were generally low throughout the season, suggesting that general practitioners may have used more general codes to record exacerbations.

To ensure that there was no dual diagnosis and that asthma and COPD were mutually exclusive, we used a coding selection exclusion process by which a patient's record was excluded from one disease cohort if it had a previous record of the other condition. In keeping with this process, smoking among our asthma cohort (24\%) was lower than that in our COPD cohort $(47 \%)$, which is comparable to previous findings. ${ }^{23}$ A recent GPRD validity study showed good accuracy in general practitioner identification of COPD and in distinguishing COPD from asthma. ${ }^{24}$

\section{Self-controlled case series method}

We chose to use the GPRD to allow us to study a large cohort of people with asthma and COPD with sufficient statistical power to investigate the safety of influenza vaccination with regard to rare clinically important outcomes. Observational studies, however, are prone to bias and confounding and a particular problem for our study was the underlying severity of airflow obstruction, since individuals with more severe disease are more likely to have exacerbations and also to be vaccinated. $^{25-27}$ Indeed, our exploration of crude outcome rates confirmed that patients who were vaccinated did have a higher incidence of outcomes. For this reason we chose to use the self-controlled case series method which compares the incidence of an outcome during specific high risk periods with other baseline periods in the same person, thus removing confounding by severity. This method also has the advantage of removing confounding by other variables that vary between individuals including medication use, social environment, sex, body mass index, smoking habit, onset age of respiratory disease, socioeconomic status, and ethnicity. ${ }^{1125}$ The lack of increase in the incidence of outcomes shortly after vaccination therefore provides strong evidence that influenza vaccination does not increase the frequency of exacerbations in this patient group.

\section{Comparison with other studies}

Our results are consistent with previous findings from clinical trials of influenza vaccine in adults, ${ }^{9-13}$ including those with respiratory illnesses. Govaert et al randomised 1806 people over 60 years of age to vaccine or placebo and found no differences in respiratory reactions between the groups; however, only $10 \%$ in each group had a lung condition. ${ }^{9}$ In a multicentre crossover trial of 2032 children and adults aged 3-64 years with asthma, no differences were found in selfreported exacerbations, including subclinical symptoms, use of asthma medications, and unscheduled health care visits for asthma. ${ }^{11}$ The same researchers found concurrent negative results after conducting a subgroup analysis of those with more severe asthma. In a smaller trial of only 255 
individuals aged 18-75 years with asthma, Nicholson et al found slight decreases in peak expiratory flow 72 hours after injection which became insignificant after exclusion of participants with colds, and concluded that the benefits of vaccination outweighed any minor risk of complications. ${ }^{13}$ Our findings are also in agreement with a population based observational study of over 20000 children with asthma, controlled completely for severity with the self-controlled case series method, which studied clinically important exacerbations over three influenza seasons. ${ }^{22}$ In the same study Kramarz et al also conducted a traditional cohort analysis and, although exacerbations were still not significantly increased, point estimates of adjusted rate ratios were inflated, demonstrating the biased results obtained even when using good proxy measures to control for severity. Few studies have specifically investigated influenza vaccination and COPD exacerbations and those available have lacked statistical power. ${ }^{15}$ When live influenza vaccine was administered to people with chronic bronchitis with airways obstruction, no adverse respiratory effects were found in two out of three placebo controlled trials, although there were fewer that 25 participants in the largest study arm of any trial and end points measured were self-reported subclinical symptoms of cough and breathlessness. ${ }^{28}$ Another partially blinded trial of only 29 subjects with COPD showed no significant difference in the number of exacerbations after live vaccine compared with placebo. ${ }^{29}$

\section{Conclusions}

Routine influenza vaccination in the general UK population of older individuals with asthma or COPD does not increase the short term incidence of adverse respiratory outcomes and the need for oral corticosteroid prescriptions. The low vaccination coverage (under $40 \%$ ) in our cohort, which is in agreement with previous reports of vaccination in high risk groups in the UK and the US, ${ }^{2}{ }^{7}$ indicates that there is much need for encouragement of vaccine uptake among this population. Promotion should occur through implementation of current guidelines that follow public health methods with proven effectiveness-such as recommendation by primary health care workers and public education ${ }^{7}$-in order to reduce the adverse impact of influenza on populations at high risk of infection.

\section{Authors' affiliations}

L J Tata, J West, R Hubbard, Division of Epidemiology and Public Health, University of Nottingham, Nottingham, UK

T Harrison, C Smith, R Hubbard, Division of Respiratory Medicine, Nottingham City Hospital, Nottingham, UK

P Farrington, Statistics, The Open University, UK

Source of funding: Junior Research Fellowship, School of Community Health Sciences, University of Nottingham

\section{REFERENCES}

1 Ahmed AH, Nicholson KG, Nguyen-van Tam JS, et al. Effectiveness of influenza vaccine in reducing hospital admissions during the 1989-90 epidemic. Epidemiol Infect 1997;118:27-33.
2 Nichol KL, Margolis KL, Wuorenma J, et al. The efficacy and cost effectiveness of vaccination against influenza among elderly persons living in the community. N Engl J Med 1994;331:778-84.

3 Ahmed AE, Nicholson KG, Nguyen-Van-Tam JS. Reduction in mortality associated with influenza vaccine during 1989-90 epidemic. Lancet 1995;346:591-5.

4 Govaert TM, Thiij CT, Masurel N, et al. The efficacy of influenza vaccination in elderly individuals. A randomized double-blind placebo-controlled trial. JAMA 1994;272:1661-5.

5 Gross PA, Hermogenes AW, Sacks HS, et al. The efficacy of influenza vaccine in elderly persons. A meta-analysis and review of the literature. Ann Intern Med 1995; 123:518-27.

6 Nicholson KG, Snacken R, Palache AM. Influenza immunization policies in Europe and the United States. Vaccine 1995;13:365-9.

7 Nguyen-Van-Tam JS, Nicholson KG. Influenza immunization; vaccine offer, request and uptake in high-risk patients during the 1991/2 season. Epidemiol Infect 1993; 111:347-55.

8 Rothbarth PH, Kempen BM, Sprenger MJ. Sense and nonsense of influenza vaccination in asthma and chronic obstructive pulmonary disease. Am J Respir Crit Care Med 1995; 151:1682-6.

9 Govaert TM, Dinant GJ, Aretz K, et al. Adverse reactions to influenza vaccine in elderly people: randomised double blind placebo controlled trial. $B M$ 1993;307:988-90.

10 Margolis KL, Nichol KL, Poland GA, et al. Frequency of adverse reactions to influenza vaccine in the elderly. A randomized, placebo-controlled trial. JAMA 1990;264:1139-41.

11 Anon. The safety of inactivated influenza vaccine in adults and children with asthma. N Engl J Med 2001;345:1529-36.

12 Stenius-Aarniala B, Huttunen JK, Pyhala R, et al. Lack of clinical exacerbations in adults with chronic asthma after immunization with killed influenza virus. Chest 1986;89:786-9.

13 Nicholson KG, Nguyen-Van-Tam JS, Ahmed AH, et al. Randomised placebocontrolled crossover trial on effect of inactivated influenza vaccine on pulmonary function in asthma. Lancet 1998;351:326-31.

14 Cates CJ, Jefferson TO, Bara Al, et al. Vaccines for preventing influenza in people with asthma (Cochrane Review). Cochrane Database Syst Rev 2000:CD000364.

15 Poole PJ, Chacko E, Wood-Baker RW, et al. Influenza vaccine for patients with chronic obstructive pulmonary disease. Cochrane Database Syst Rev 2000:CD002733.

16 EPIC. The general practice research database: a guide for researchers. 2001.

17 Lawson DH, Sherman V, Hollowell J. The General Practice Research Database. Scientific and Ethical Advisory Group. Q J Med 1998;91:445-52.

18 Garcia Rodriguez LA, Perez Gutthann S. Use of the UK General Practice Research Database for pharmacoepidemiology. Br J Clin Pharmacol 1998:45:419-25.

19 Hansell A, Hollowell J, Nichols T, et al. Use of the General Practice Research Database (GPRD) for respiratory epidemiology: a comparison with the 4th Morbidity Survey in General Practice (MSGP4). Thorax 1999;54:413-9.

20 Farrington $P$, Pugh S, Colville A, et al. A new method for active surveillance of adverse events from diphtheria/tetanus/pertussis and measles/mumps/ rubella vaccines. Lancet 1995;345:567-9.

21 Farrington CP, Nash J, Miller E. Case series analysis of adverse reactions to vaccines: a comparative evaluation. Am J Epidemiol 1996;143:1 165-73; erratum 1998; 147:93.

22 Kramarz P, DeStefano F, Gargiullo PM, et al. Does influenza vaccination exacerbate asthma? Analysis of a large cohort of children with asthma. Vaccine Safety Datalink Team. Arch Fam Med 2000;9:617-23.

23 Wijnhoven HA, Kriegsman DM, Hesselink AE, et al. Determinants of different dimensions of disease severity in asthma and COPD: pulmonary function and health-related quality of life. Chest 2001;119:1034-42.

24 Soriano JB, Maier WC, Visick G, et al. Validation of general practitionerdiagnosed COPD in the UK General Practice Research Database. Eur J Epidemiol 2001;17:1075-80.

25 Kramarz P, DeStefano F, Gargiullo PM, et al. Influenza vaccination in children with asthma in health maintenance organizations. Vaccine Safety Datalink Team. Vaccine 2000;18:2288-94.

26 Fine PE, Chen RT. Confounding in studies of adverse reactions to vaccines. Am J Epidemiol 1992;136:121-35.

27 Hak $E$, Hoes AW, Grobbee DE, et al. Conventional influenza vaccination is not associated with complications in working-age patients with asthma or chronic obstructive pulmonary disease. Am J Epidemiol 2003; 157:692-700.

28 MRC Advisory Group on Pulmonary Function Tests in Relation to Live Influenza Vaccines. Trials of live attenuated influenza virus vaccine in patients with chronic obstructive airways disease. Br J Dis Chest 1984;78:236-47.

29 Gorse GJ, Otto EE, Daughaday CC, et al. Influenza virus vaccination of patients with chronic lung disease. Chest 1997;1 12:1221-33. 